\title{
Person-centered integrated approach in an advanced heart failure clinic
}

\author{
Kira Stellato ${ }^{1 *}$, Franco Humar ${ }^{1}$, Cristina Montesi ${ }^{1}$, Donatella Radini', Gioia Sola', Nadia Zeriali', \\ Franca Frigo', Gianfranco Sinagra ${ }^{2}$, Andrea Di Lenarda ${ }^{1}$ \\ ${ }^{1}$ Cardiovascular Center, Health Authority $n^{\circ} 1$ and University of Trieste, Trieste, Italy \\ ${ }^{2}$ Cardiovascular Department, "Ospedali Riuniti" and University of Trieste, Trieste, Italy
}

Goal: Cardiovascular disease (CVD) constitutes epidemiological, clinical and social emergency and requires an effective and efficient person and people-centered integrated interventions to empower patients, increase adherence and improve clinical-psycho-social outcome. This interdisciplinary, integrated approach requires holistically attending to the person's needs both through the supportive and the palliative stages of heart failure.

Material and Methods: In 2013, an Advanced Heart Failure Clinic (AHFC) was activated within the Outpatient Cardiovascular Department in Trieste. AHFC is made up of an interdisciplinary team (2 cardiologists, an internist, a psychologist, and 4 dedicated nurses) who collaborate with GPs and healthcare professionals from the four District teams which make up the Province of Trieste. The goal is to provide person-centered, integrated supportive and palliative care to patients with an advanced heart failure (NYHA III-IV) after the discharge from hospital/residential facility, or to those requiring longitudinal monitoring (both as outpatients or at their homes). A Person-Centered Psycho-Social Wellbeing Questionnaire (PCPSWQ) was developed to investigate personal, social and healthcare well-being as well as the knowledge of the disease and the level of adherence.

Received: $1^{\text {st }}$ May 2014

${ }^{*}$ Address for correspondence: Cardiovascular Center, Health Authorithy $n^{\circ} 1$ and University of Trieste, Trieste, Italy

E-mail: ks@menteebenessere.it
Clinical and instrumental data were retrieved from the Cardionet $^{\circledR}$ electronic registry of Trieste Cardiovascular Center.

Results: In 2013, AHFC made a total of 479 visits (173 patients; $68 \%$ males; mean age 76 $\pm 9 ; 68 \%$ NYHA 3-4; $92 \%$ in polypharmacological therapy; $45 \%$ Charlson index $>5.52$ questionnaires were administered to 46 patients (38 males, age $74.9 \pm$, years of education $8.5 ; 13$ living alone or with a disabled relative). The structured questionnaires were integrated by 44 narrative interviews. $85 \%$ of the patients expressed satisfaction for their relationship with their GP/specialist. However, $45 \%$ expressed little knowledge or awareness of their disease. $78 \%$ stated optimal adherence to the therapy, but only $55 \%$ follow an adequate nutrition and lifestyle regimen.

Conclusions: These preliminary results highlight the importance of an integrated, person-centered beehive approach to identify subjective and objective needs and develop multimodal communication instruments to increase patient's knowledge, awareness, self empowerment and self-management capabilities. Person-centered, integrated care needs to be redesigned through a more thorough investigation of patients' clinical, psychological and social dimensions and interdisciplinary teams must develop and implement integrated assessments to prevent fragmentation of the person and care provided to the person.

KEYWORDS: integrated healthcare, person-centered approach, multidisciplinary care,

muldimensional evaluation, heart failure.

CITATION: Cardiol Croat. 2014;9(5-6):219. 